\title{
Reliability of a Fly Motion-Sensitive Neuron Depends on Stimulus Parameters
}

\author{
Anne-Kathrin Warzecha, Jutta Kretzberg, and Martin Egelhaaf \\ Lehrstuhl für Neurobiologie, Fakultät für Biologie, Universität Bielefeld, D-33501 Bielefeld, Germany
}

The variability of responses of sensory neurons constrains how reliably animals can respond to stimuli in the outside world. We show for a motion-sensitive visual interneuron of the fly that the variability of spike trains depends on the properties of the motion stimulus, although differently for different stimulus parameters. (1) The spike count variances of responses to constant and to dynamic stimuli lie in the same range. (2) With increasing stimulus size, the variance may slightly decrease. (3) Increasing pattern contrast reduces the variance considerably. For all stimulus conditions, the spike count variance is much smaller than the mean spike count and does not depend much on the mean activity apart from very low activities. Using a model of spike generation, we analyzed how the spike count variance depends on the

All information an animal is able to acquire about its environment is contained in the activity of its nerve cells. Therefore, the variability of neuronal responses constrains how reliably stimuli can be perceived or responded to. In cortical visual interneurons, spike count variances have been found to be in the order of the mean spike count (Tolhurst et al., 1983; Vogels et al., 1989; Britten et al., 1993; Barberini et al., 2000). Smaller spike count variances have been observed at more peripheral stages of the vertebrate visual system (Levine et al., 1988, 1992; Berry et al., 1997; Reinagel and Reid, 2000) and in motion-sensitive neurons of the fly (de Ruyter van Steveninck et al., 1997; Warzecha and Egelhaaf, 1999).

In visual neurons, a given activity can usually be elicited by various combinations of stimulus parameters. For example, the output of motion-sensitive visual interneurons may depend on the size, the contrast, and the velocity of the stimulus. An extended pattern with a small contrast may elicit the same response as a smaller pattern with a high contrast. Moreover, stimuli with temporally modulated velocity might lead, at a certain response phase, to the same response level as a constant velocity stimulus. Because in these situations the spatiotemporal activity distribution across the synaptic inputs of the neuron may differ considerably, the variability in the resulting responses may also differ. Apart from studies that explicitly investigated whether the stimulus dynamics affects neuronal variability (Berry et al., 1997; de Ruyter van Steveninck et al., 1997; Buračas et al., 1998; Warzecha and Egelhaaf, 1999), there are only few studies on the dependence of neuronal variability on the stimulus conditions (Dijk and Ringo, 1987; Croner et al., 1993).

Therefore, we analyze the stimulus dependence of the acrosstrial variance of the spike activity of the H1 neuron in the fly visual system. The H1 neuron, as well as other so-called tangential cells

\footnotetext{
Received May 17, 2000; revised Sept. 7, 2000; accepted Sept. 11, 2000.

This study was supported by the Deutsche Forschungsgemeinschaft and the Studienstiftung des Deutschen Volkes. We thank Judith Eikermann for help with the electrophysiological experiments. We are grateful to Katja Karmeier, Roland Kern, Holger Krapp, and Rafael Kurtz for reading this manuscript and helpful discussions.

Correspondence should be addressed to Dr. Anne-Kathrin Warzecha, Lehrstuh für Neurobiologie, Fakultät für Biologie, Universität Bielefeld, Postfach 1001 31, D-33501 Bielefeld, Germany. E-mail: ak.warzecha@biologie.uni-bielefeld.de. Copyright (C) 2000 Society for Neuroscience $0270-6474 / 00 / 208886-11 \$ 15.00 / 0$
}

membrane potential noise and the deterministic membrane potential fluctuations at the spike initiation zone of the neuron. In a physiologically plausible range, the variance is affected only weakly by changes in the dynamics or the amplitude of the deterministic membrane potential fluctuations. In contrast, the amplitude and dynamics of the membrane potential noise strongly influence the spike count variance. The membrane potential noise underlying the variability of the spike responses in the motion-sensitive neuron is concluded to be affected considerably by the contrast of the stimulus but by neither its dynamics nor its size.

Key words: reliability; variability; motion vision; neural coding; spike generation; model; fly

(TCs), of the fly have been widely used for investigating neuronal processing of visual motion information (for review, see Hausen and Egelhaaf, 1989; Egelhaaf and Borst, 1993; Egelhaaf and Warzecha, 1999) and, in particular, the reliability of neural coding (de Ruyter van Steveninck and Bialek, 1988, 1995; de Ruyter van Steveninck et al., 1997; Haag and Borst, 1997; Warzecha and Egelhaaf, 1997, 1999; Warzecha et al., 1998). Our electrophysiological analysis is supplemented by model simulations, which will form the basis for interpreting the experimental results. The simulations are based on a phenomenological model of spike generation that was adjusted to account for the response properties of the H1 neuron (Kretzberg et al., 2000). In the simulations, the postsynaptic membrane potential is varied systematically to analyze the determinants of the resulting spike responses. The spike count variance will be shown not to be determined unambiguously by the mean spike activity but to depend also on the visual input that induces a given activity level. Irrespective of this stimulus dependence, the spike count variance will be shown to be considerably smaller than the mean spike count for all stimulus conditions.

\section{MATERIALS AND METHODS}

Preparation and electrophysiology. For electrophysiological experiments, female blowflies of the genus Calliphora were obtained from our laboratory stocks. To avoid inbreeding, the stocks were regularly refreshed by animals caught outside. The animals were dissected as described previously (Warzecha and Egelhaaf, 1997). The experiments were performed at room temperature $\left(19-22^{\circ} \mathrm{C}\right)$. Experiments were done in compliance with institutional guidelines and those of the Society for Neuroscience.

The H1 neuron can be identified unambiguously on the basis of its preferred direction of motion and the location of its extended output region (Eckert, 1980; Hausen, 1981). The activity of the H1 neuron was recorded extracellularly with tungsten electrodes in its output region. The electrode tips were sharpened electrolytically and insulated with varnish. They had resistances between 2 and $8 \mathrm{M} \Omega$. Recorded signals were processed by standard electrophysiological equipment. Spikes were converted into pulses of fixed height and duration, which were fed at a rate of $1 \mathrm{kHz}$ into a personal computer through an analog-to-digital converter of an input-output card (2801A; Data Translation Inc., Marlboro, MA). Only extremely well isolated $\mathrm{H} 1$ signals were recorded, so that our data were free of noise introduced at the level of data acquisition. The programs for data acquisition were written in ASYST (Keithley Instruments, Cleveland, $\mathrm{OH})$.

Visual stimulation. Moving square wave gratings were used as stimuli (spatial wavelength, $18^{\circ}$; mean luminance, $7.9 \mathrm{~cd} / \mathrm{m}^{2}$; contrast as specified in figure legends) and displayed on a cathode ray tube (model 608; 
Tektronix, Wilsonville, OR) at a frame rate of $183 \mathrm{~Hz}$ by an image synthesizer (Picasso; Innisfree Inc., Cambridge, MA). In previous investigations, it was ensured that spikes do not time lock to this frame rate (Warzecha et al., 1998; Warzecha and Egelhaaf, 1999). The image synthesizer was controlled by a personal computer. The center of the monitor was at an azimuth/elevation of $45^{\circ} / 0^{\circ}$, with $0^{\circ} / 0^{\circ}$ referring to the frontal midline of the fly. The front edge of the monitor screen was at an azimuthal position of $0^{\circ}$. The horizontal extent of the stimulus pattern was $90^{\circ}$, and its vertical extent was varied and will be given in the figure legends. Irrespective of the vertical extent, the vertical position of the pattern was always centered at an elevation of $0^{\circ}$ in the receptive field of the $\mathrm{H} 1$ neuron. Data acquisition was started with the frame synchronization signal of the image synthesizer. Four different data sets were obtained by varying the vertical extent of the stimulus pattern, its contrast, and/or its velocity. For each data set, a different sample of flies was used. For each fly, all visual stimuli used to obtain the particular data set were presented in a pseudorandom order, so that each stimulus was presented once before the next sequence started. Two types of pattern motion dynamics were used: constant and randomly fluctuating velocity. For constant velocity stimuli, the pattern was moved in the preferred direction of the cell for $2.5 \mathrm{sec}$. To obtain a dynamic velocity stimulus, white-noise velocity fluctuations were generated according to a gaussian distribution. The resulting velocity trace was low-pass filtered with a cutoff at $80 \mathrm{~Hz}$ to avoid aliasing caused by the frame rate limit. After low-pass filtering, the SD of the velocity trace was $0.12 \% \mathrm{msec}$. For the dynamic velocity fluctuations, pattern motion lasted for $5 \mathrm{sec}$. Presentation of motion stimuli was interrupted by an interval of 6.5 sec. During this interval, the stimulus pattern was homogeneous with a luminance of $7.9 \mathrm{~cd} / \mathrm{m}^{2}$ corresponding to the mean luminance of all moving stimuli that were used in the experiments. For the experiments with stimuli of variable vertical extent, the mean luminance was presented in all parts of the screen that were not covered by the motion stimulus.

Data evaluation. For constant velocity stimuli, only $1000 \mathrm{msec}$ of the response starting $1500 \mathrm{msec}$ after the onset of motion were evaluated to analyze primarily the steady-state response instead of the onset transients. For dynamic velocity stimuli, $4900 \mathrm{msec}$ starting $100 \mathrm{msec}$ after the onset of motion were evaluated. For each stimulus condition and each cell, 40-60 consecutive trials were taken for quantitative analysis.

The mean spike count as well as the spike count variance were determined within time windows of variable size. The shortest time window used was $20 \mathrm{msec}$ because shorter time windows lead to results that hardly reflect the variability of the responses (Warzecha and Egelhaaf, 1999). On the other hand, if the time windows are too long, they average out activity modulations as may be elicited by dynamic motion stimuli. Because the dependence of the variance on the mean activity of the cell may differ for different time windows, results will be displayed for time windows of 20 and $100 \mathrm{msec}$ size. We thus cover a large range of time windows used in previous studies on the variability of the fly H1 neuron (de Ruyter van Steveninck et al., 1997; Warzecha and Egelhaaf, 1999). The mean spike count and the corresponding spike count variance were evaluated across trials in consecutive time windows that were shifted by $10 \mathrm{msec}$. Hence, consecutive 20 or $100 \mathrm{msec}$ time windows overlapped by 10 or $90 \mathrm{msec}$, respectively. This evaluation was done separately for each cell, each stimulus condition, and each size of the time window. For dynamical velocity stimulation, the mean spike count was assigned to activity classes with a width of either 0.4 spikes/20 msec window or 2 spikes/100 msec window. Variances of each cell were averaged if the corresponding mean spike count fell into the same activity class. The variances associated with a given activity class, pattern size, and time window were averaged, irrespective of the response phase during which the activity was attained. These values were then averaged over the cells contributing to one data set. Only those activity classes and corresponding variances will be shown to which at least four cells contributed.

During constant stimulation, the activity stays almost constant and modulates only weakly with the temporal frequency of pattern motion. The activity thus spreads only over very few activity classes. The variances were therefore not averaged within different activity classes. Only a single variance value was determined together with the corresponding mean spike count for each stimulus condition and cell. These variances and mean spike counts were then averaged over all cells of a data set.

The variance of the responses strongly depends on whether the neuronal activity shows a systematic trend over the recording period. We selected by the following procedure only those data for further analysis that did not show a strong trend. The mean activity during stimulation with any of the motion stimuli presented to a given cell was not allowed to change by more than $1 \mathrm{spike} / \mathrm{sec}$ and per trial, as judged from a regression line through the mean activities plotted over the number of trials. Therefore, 13 of 52 cells had to be discarded.

Model simulations. To investigate the determinants of the spike count variance, we used a phenomenological threshold model that transforms time-dependent membrane potential fluctuations into sequences of action potentials. These sequences will be compared with the spike responses of the H1 cell. The details of the model have been described previously (Kretzberg et al., 2000). In brief, for every time step, the membrane potential is compared with a spike threshold that depends on both the time elapsed since the previous spike occurred and the temporal changes of the membrane potential. A spike is fired when the membrane potential crosses threshold. The threshold is calculated according to the following equation:

$$
\theta\left(t_{i}\right)=\left\{\begin{array}{ll}
\infty & \text { if } s \leq \gamma^{\text {ref }} \\
\theta_{0}+\eta(s)+\rho\left(t_{i}\right) & \text { if } s>\gamma^{\text {ref }}
\end{array}\right\}
$$

with $t_{i}$ indicating time step, $s$ indicating time elapsed since the previous spike, $\gamma^{\text {ref }}$ indicating absolute refractory period, and $\theta_{0}$ indicating constant basis threshold.

$$
\eta(s)=\frac{\eta_{0}}{s-\gamma^{\mathrm{ref}}}
$$

is the influence of the relative refractoriness with weight constant $\eta_{0}$.

$$
\rho\left(t_{i}\right)=-\frac{\rho_{0}}{T} \cdot \sum_{j=1}^{T} \frac{1}{j} \cdot\left(U\left(t_{i}\right)-U\left(t_{i-j}\right)\right)
$$

is the influence of the membrane potential changes within the last $T$ data points, with weight constant $\rho_{0}$ and membrane potential $U\left(t_{i}\right)$.

For a constant membrane potential, the term $\eta(s)$ causes the threshold to decrease to the constant value $\theta_{0}$ after the absolute refractory period $\gamma^{\text {ref }}$. When the membrane potential varies, the resulting threshold is influenced by the term $\rho(t)$. It represents the weighted and sign-inverted sum of the slopes between the last $T$ membrane potential values and the reference potential $U\left(t_{i}\right)$ at time $t_{i}$. The threshold decreases while the membrane potential depolarizes, and it rises while $U(t)$ hyperpolarizes. The steeper the membrane potential rises or falls, the more the threshold is influenced by the term $\rho(t)$. This term has been included in the model, because fast depolarizing changes in the membrane potential are generally found to be more effective in eliciting a spike than slow ones (Johnston and Wu, 1995; Azouz and Gray, 2000).

The model cell was fed by membrane potential fluctuations as they were elicited by motion stimuli with random velocity fluctuations in a fly TC (HS cell). This neuron has a similar input organization as the $\mathrm{H} 1$ neuron and responds to motion stimulation with pronounced membrane potential changes, which are assumed to closely reflect the pooled postsynaptic potentials of many retinotopically organized motion-sensitive elements (for a detailed discussion of this aspect, see Kretzberg et al. 2000). These potentials are assumed to be similar to the postsynaptic potentials of the H1 neuron. Methodological difficulties render it impracticable to directly record the postsynaptic potentials of the $\mathrm{H} 1$ neuron.

The input to the model of spike generation consisted of two components, a deterministic and a stochastic one. (1) The deterministic component of the membrane potential was derived from the time-dependent membrane potential of the HS cell averaged over 100 responses to identical dynamical motion stimulation. The averaging was assumed to eliminate stochastic membrane potential fluctuations. The average was sign-inverted to account for the opposite preferred directions of the HS and the H1 cell and taken to represent the stimulus-induced response component. The response traces of the HS cell that formed the basis of the model simulations were obtained in a previous study under one given stimulus condition (Warzecha et al., 1998, their Fig. 3).

(2) The stochastic component of the membrane potential was computed individually for each trial as a series of gaussian distributed random numbers that was low-pass filtered. A gaussian probability distribution fitted quite well the experimentally determined distribution of the membrane potential noise. By adopting the SD of the noise from experimental data and by temporally filtering the series of random numbers, the power spectrum of the stochastic component was fitted to the power spectrum of the experimentally determined membrane potential noise of the HS cell (Warzecha et al., 1998; Kretzberg et al., 2000). The terms "stochastic component" and "membrane potential noise" will both be used in the following as synonyms.

The model simulations allowed us to systematically vary the deterministic and the stochastic response components separately from each other. This was done by scaling the amplitude of either component with a factor or by stretching their time scale (for details, see Results and figure legends). Both components were added and then fed into the spike generation mechanism. If not specified otherwise, 500 different stochastic sequences with the same statistical properties were used for every analysis. Each sequence lasted for 2960 msec.

The model parameters were adjusted so that the resulting spike trains fit, on average, the spike trains of the $\mathrm{H} 1$ cell when stimulated with the same dynamic motion stimuli as the HS cell (for details, see Kretzberg et al., 2000 ). With the parameter sets obtained in this way, the relevant features of the $\mathrm{H} 1$ cell response, such as the time-dependent spike frequency histogram, as well as its highly correlated activity with another spiking neuron that receives partly the same input can be readily explained (Kretzberg et al., 2000). The model simulations presented here were done with five parameter sets, identical to those used in a previous paper (Kretzberg et al. 2000). All parameter sets lead to qualitatively the same results. The data that are shown in Figures $6-9$ were obtained with the following parameters: $\gamma^{\mathrm{ref}}=2 \mathrm{msec}, \theta_{0}=1 \mathrm{mV}, \eta_{0}=20 \mathrm{msec} \cdot \mathrm{mV}, \rho_{0}=$ 3.75 , and $T=3$ data points. A temporal resolution of $2.7 \mathrm{kHz}$ was used for the model simulations. 

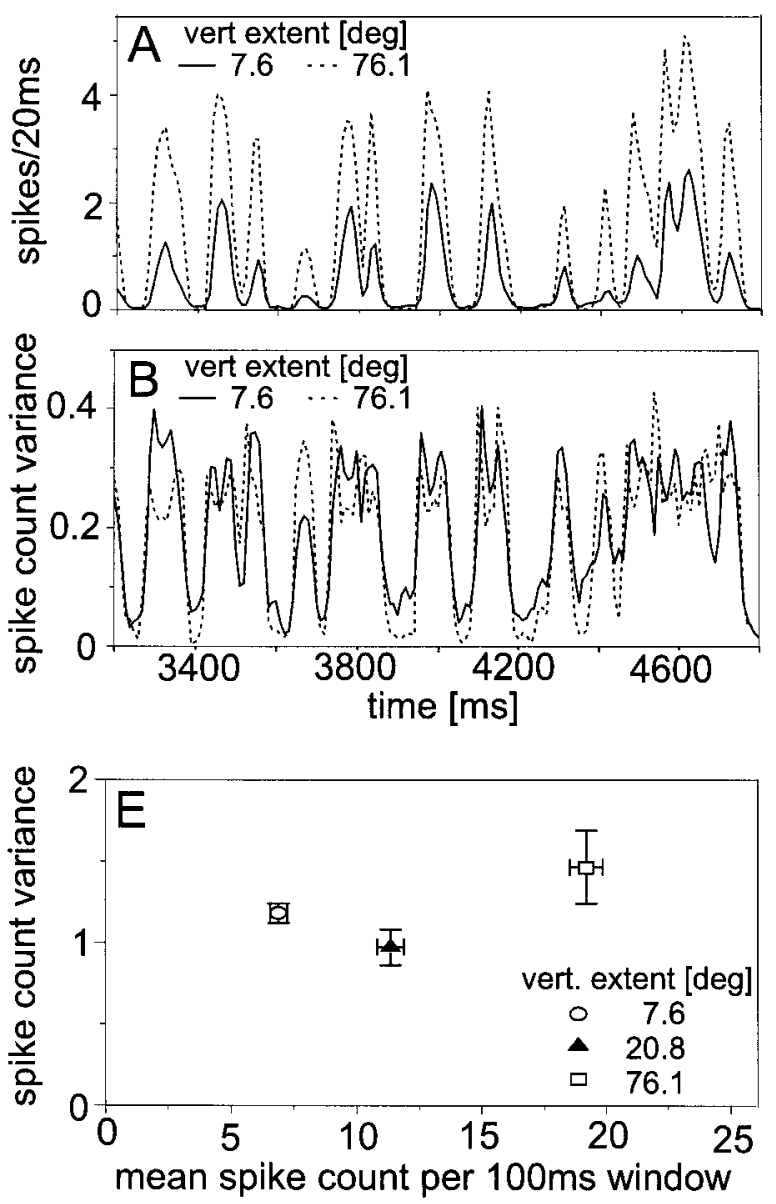
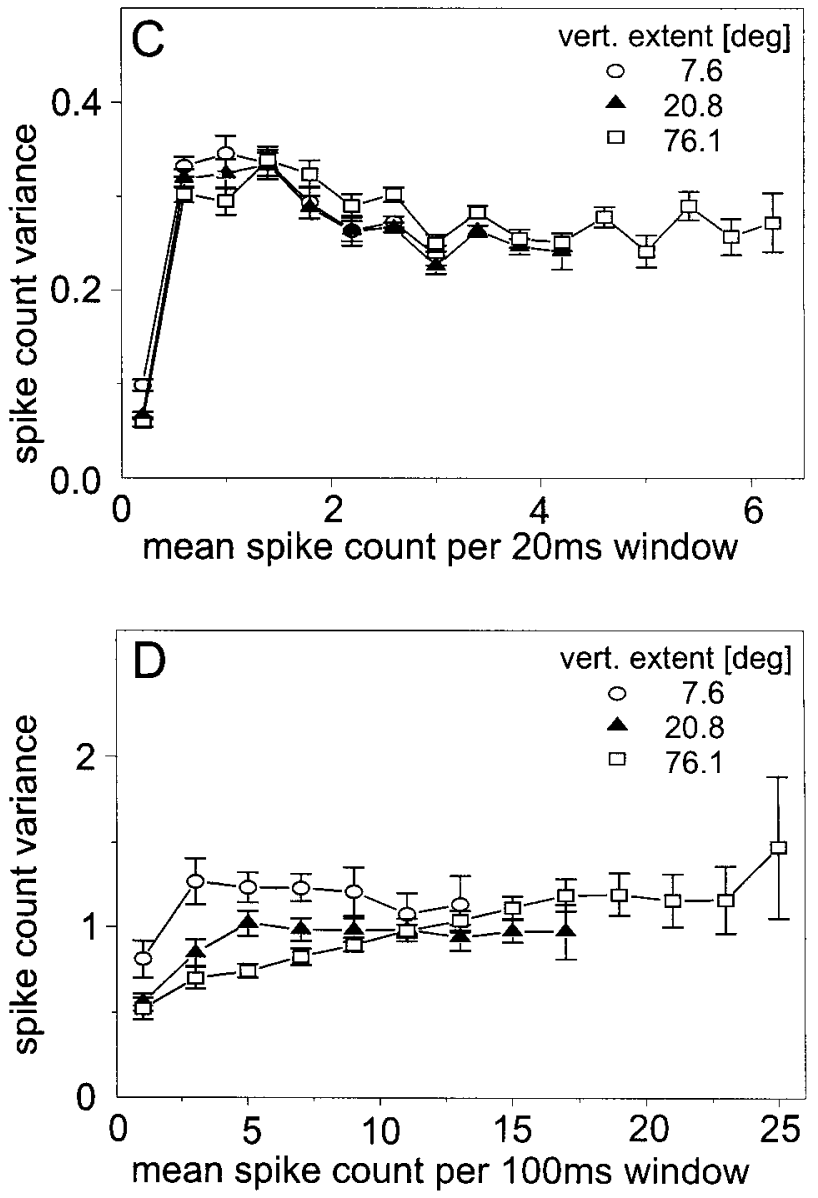

Figure 1. Response variability of the $\mathrm{H} 1$ neuron obtained for the motion of stimulus patterns with variable size. See insets for the vertical extent of the pattern. Altogether, 457 individual response traces of eight $\mathrm{H} 1$ cells were analyzed. The mean resting activity was $9.1 \mathrm{spikes} / \mathrm{sec}$. Pattern contrast, $20 \%$. $A$, Section of the mean time course of responses to band-limited white-noise velocity fluctuations. Spikes were counted in each trial within consecutive time windows of $20 \mathrm{msec}$ time-locked to the onset of motion. Consecutive time windows overlapped by $10 \mathrm{msec}$. Spike counts in corresponding time bins were averaged across trials. Time 0 denotes the onset of the stimulus. B, Mean time course of the across-trial variance of the spike count obtained within the same section of $20 \mathrm{msec}$ time windows. $C$, Spike count variance as a function of the mean spike count within 20 msec time windows obtained for band-limited white-noise velocity fluctuations (see Materials and Methods). $D$, Spike count variance as a function of the mean spike count within 100 msec time windows obtained for band-limited white-noise velocity fluctuations. $E$, Spike count variance as a function of the mean spike count within 100 msec time windows obtained for constant velocity stimulation. The temporal frequency of pattern motion amounted to $2 \mathrm{~Hz}$. $C-E$, Error bars denote SEMs across trials. $C, D$, Although these experiments were made on eight cells, a variable number of cells $(4-8)$ contributed to each data point because not every cell covered the entire activity range.

The simulated data were evaluated in the same way as the experimental ones. The model simulations and all evaluation routines were implemented in Matlab 5.3 (The MathWorks Inc., Natick, MA).

\section{RESULTS}

\section{Experimental analysis of stimulus dependence of the variance}

The H1 neuron integrates the output signals of many local motionsensitive elements with basically the same direction selectivity. Therefore, it responds selectively to motion in a particular direction in large parts of the visual field of one eye. It increases its spike activity above the resting level during back-to-front motion ("preferred direction") and decreases its spike rate during front-to-back motion ("null direction"). Because the spontaneous firing rate of the $\mathrm{H} 1$ neuron is low, the cell usually stops firing during null direction motion. When stimulated with random velocity fluctuations, the spike frequency of the $\mathrm{H} 1$ cell is modulated in time. The time course of these response modulations follows to some extent the velocity fluctuations, although it is not proportional to the velocity, in particular when the direction of pattern velocity changes rapidly (Egelhaaf and Reichardt, 1987; Haag and Borst, 1997) (for review, see Warzecha and Egelhaaf, 2000).

\section{Variation of pattern size}

Spike frequency histograms of responses to the same random velocity fluctuations of two patterns of different size are shown in Figure $1 A$. The histograms were determined with time windows of 20 msec. The response amplitude decreases with decreasing pattern size. Nonetheless, the time course of the response is very similar for the different pattern sizes (Fig. $1 A$, compare dashed lines, solid lines). Analogous to the mean spike count, the across-trial variance in the spike count can be plotted as a function of time (Fig. 1B). Here the same $20 \mathrm{msec}$ time windows were used as for the spike frequency histograms in Figure $1 A$. The variance is not constant during dynamic velocity stimulation but rather modulates in time. The modulations follow, at least to some extent, the fluctuations of the mean spike count. Nonetheless, the large differences in the amplitudes of the time-dependent spike count obtained with small and large stimulus patterns (Fig. $1 A$ ) are not reflected in corresponding differences in the time-dependent variance profiles (Fig. $1 B)$. Rather, the variances obtained with the different pattern sizes are quite similar. The data were further evaluated by determining the dependence of the across-trial variance on the mean spike count. For the $20 \mathrm{msec}$ time window, the spike count variance is small at very low activities compared with the variance obtained in 

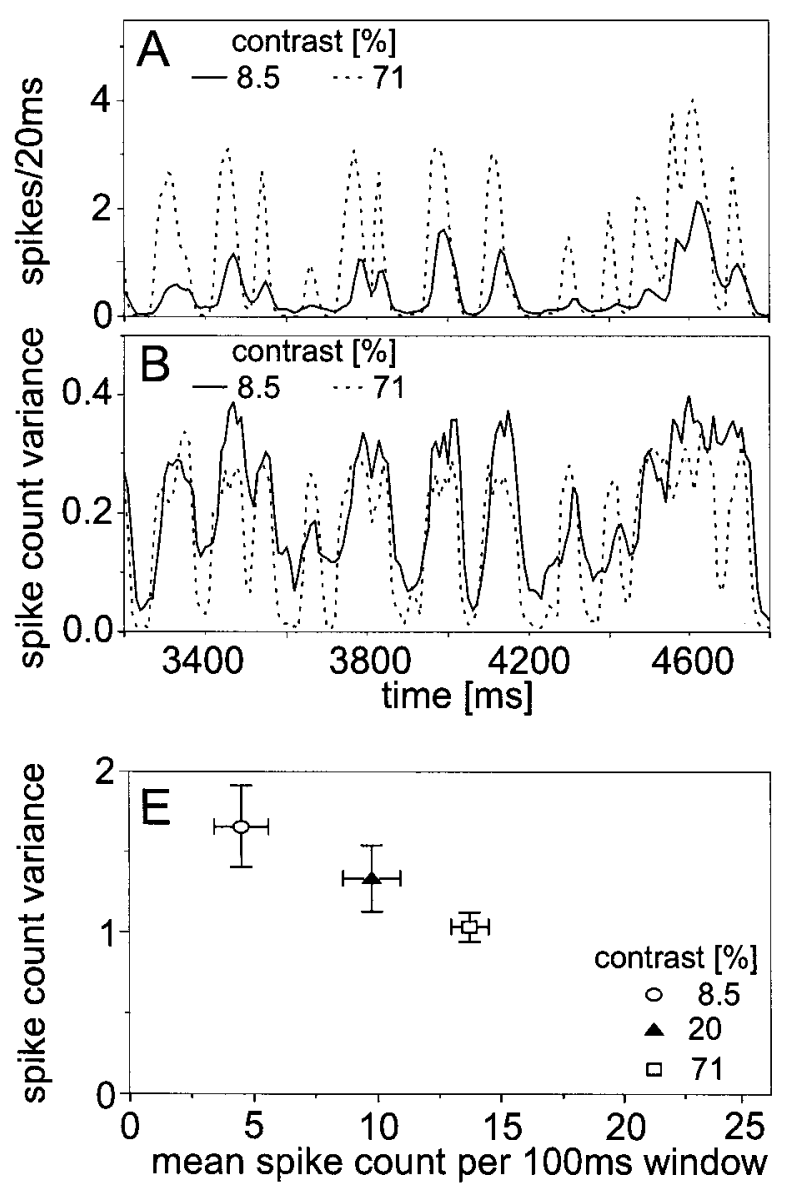
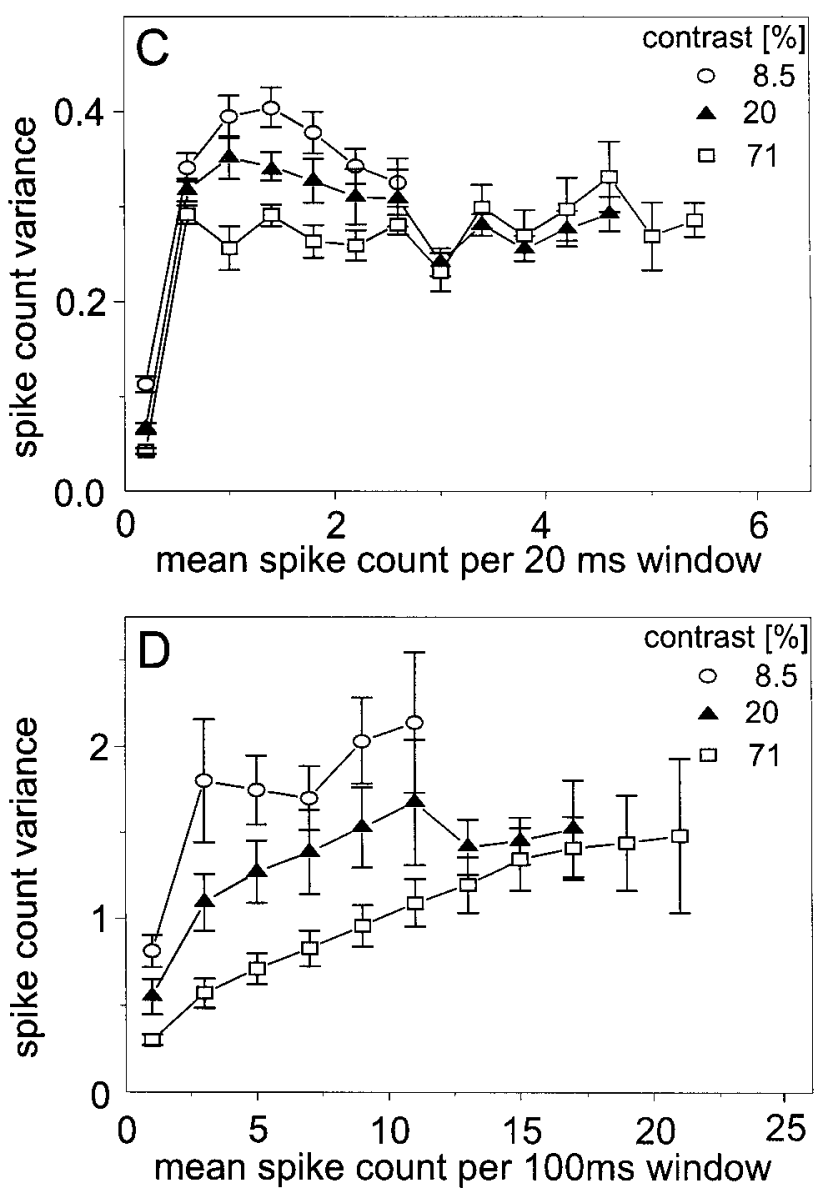

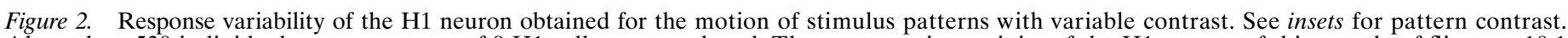

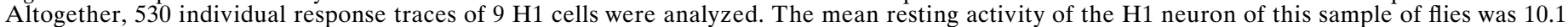

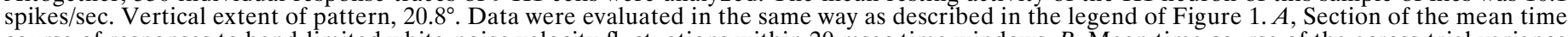

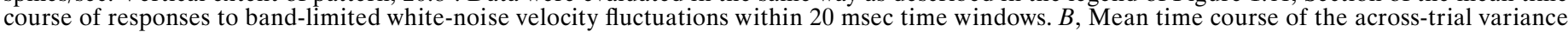

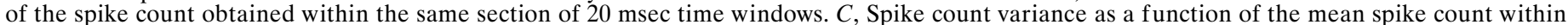

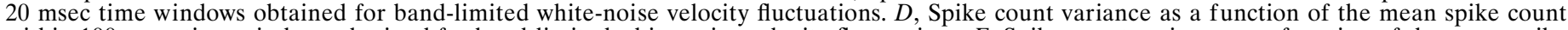

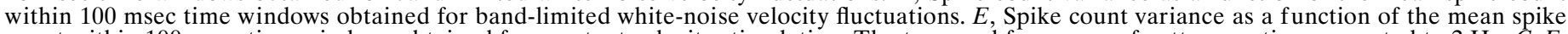

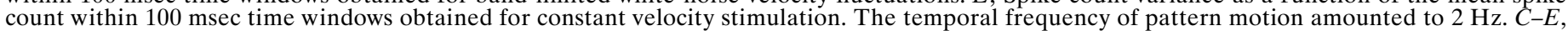
Error bars denote SEMs across cells. $C, D$, Between four and nine cells contributed to each data point.

higher activity classes (Fig. $1 C$ ). For activities above $\sim 0.6$ spikes $/ 20$ msec window (i.e., 30 spikes/sec), the variance does not increase further. Within a given activity class, the variances do not much differ for stimulus patterns of different size (Fig. 1C). In contrast, when calculated within $100 \mathrm{msec}$ time windows, there are slight differences in the variances of responses elicited by stimuli of different size. In the low-activity range, the variance is smallest for the largest stimulus pattern (Fig. 1D). For the largest pattern, the variance increases slightly with increasing mean activity. For smaller patterns, such an increase can only be observed in the low-activity range. Overall, the spike count variance does not strongly depend on the activity of the neuron. The spike count variances obtained when the H1 neuron was activated by constant velocity motion were in the same range as the ones elicited with the same pattern moving at continually changing velocities (Fig. 1, compare $D, E)$.

\section{Variation of pattern contrast}

Changing the contrast of the pattern does not much alter the time course of the response but influences mainly the response amplitude (Fig. 2A). The time-varying variance of responses to the high-contrast pattern assumes, for most of the time, slightly lower values than the variance of responses to the low-contrast stimulus (Fig. $2 B$ ). When plotting the variance as a function of the mean activity, it becomes evident that the variance differs for different stimulus contrasts, at least in the activity range of up to 2.6 spikes/20 msec time window (i.e., up to $130 \mathrm{spikes} / \mathrm{sec}$ ) (Fig. 2C). For a given activity class, higher contrasts lead to smaller variances (Fig. 2C). This difference is more pronounced when the variance is evaluated in $100 \mathrm{msec}$ than when $20 \mathrm{msec}$ time windows are used (Fig. 2, compare $C, D$ ). Hence, pattern size and pattern contrast seem to affect the spike count variance in the $\mathrm{H} 1$ cell in a different way. The variances obtained when the velocity of pattern motion was constant are in the same range as those obtained with randomly fluctuating velocities for all three pattern contrasts tested (Fig. 2, compare $D, E)$.

\section{Variation of the velocity of pattern motion}

The steady-state response amplitude of the H1 cell to constant velocity motion first increases with increasing velocity, reaches an optimum, and then declines again (Egelhaaf and Borst, 1993b). In the tested velocity range, the largest responses were obtained for small velocities (Fig. 3). With increasing activity, the variance initially increases and then decreases again. Hence, when the velocity of a stimulus is changed to alter stimulus strength, the spike count variance does not monotonically increase with increasing activity of the cell. As is the case for the experiments in which pattern size or contrast were varied, the spike count variance stays much smaller than the mean spike count. 


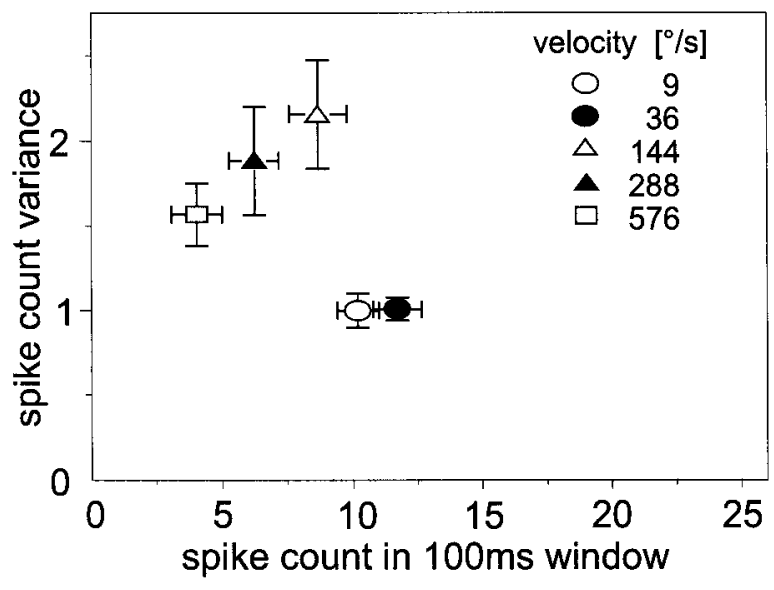

Figure 3. Response variability of the $\mathrm{H} 1$ neuron to constant velocity stimuli covering a range from 9 to $576^{\circ} / \mathrm{sec}$. The vertical extent of the pattern was $20.8^{\circ}$, and its contrast amounted to $20 \%$. Altogether, 460 individual response traces of eight $\mathrm{H} 1$ cells were analyzed. The mean resting activity was 15.3 spikes/sec. Error bars denote SEMs across eight cells.

\section{Variation of pattern contrast and pattern size}

In the next step of the analysis, we compared the variances obtained for different stimulus conditions that were chosen to lead with very similar spike frequency histograms. One stimulus pattern had a larger vertical extent but a lower contrast than the other. Both patterns were moved with the same velocity profile. The resulting spike frequency histograms (Fig. 4A) were at least much more similar than the spike frequency histograms that were obtained when either only pattern size (Fig. $1 A$ ) or pattern contrast (Fig. $2 A$ ) were varied. Moreover, both patterns led also to nearly the same mean response amplitude when they were moved at a constant velocity ( $p>0.5 ; t$ test) (Fig. $4 E)$. Despite the close correspondence of both the time course and the mean amplitude of the responses, the spike count variance across trials was significantly larger for the large low-contrast pattern than for the smaller high-contrast pattern (Fig. $4 C-E$, see figure legend for statistical details).

The results of Figure 4 go beyond those shown in Figures 1 and 2. In Figure 4, different spike count variances were obtained, although for each time interval almost the same response amplitudes were induced by the different stimuli. Hence, even for a given mean spike count and almost the same temporal profile of the responses, the spike count variance may greatly differ. Because pattern size was found to influence the spike count variability only little, it is concluded that the spike count variance is mainly influenced by pattern contrast, being largest for low-contrast stimuli.

\section{Variability between different cells}

There is a large interindividual variability in the spike count variances. Even for a given stimulus condition they may differ by a factor of up to $\sim 3.5$ independent of stimulus dynamics. As a consequence of the large interindividual variability of the spike count variance, a quantitative comparison between different data sets is problematic, at least if these are not very large. For example, the data presented in Figures 1 and 2 were each obtained from the individually identifiable $\mathrm{H} 1$ cell in a different sample of either 8 or 9 flies, respectively. One dynamical and one constant motion stimulus were the same for both data sets. Nonetheless, the corresponding data points shown in Figures $1 C-E$ and $2 C-E$ (filled triangles) show considerable quantitative differences. This variability of the response properties between different animals and data sets underlines the importance to restrict quantitative comparisons to responses obtained from the same sample of cells.

On average, variances of responses to constant and to dynamical stimuli lie in the same range, although there are cells for which the variance of responses to constant stimuli is approximately twice as large as that of responses to dynamical stimuli and vice versa (Fig. 5). There is no obvious relationship between the ratio of the variances of responses to dynamical and constant stimuli on the mean activity. Variances obtained with constant and those obtained with dynamical stimuli do not covary for any stimulus configuration used in the present study ( $t$ test, $p>0.05,8 \leq \mathrm{N} \leq 14$ ).

\section{Relationship between membrane potential fluctuations and spike count variability}

The experimental analysis led to two major results that need to be further investigated. (1) Apart from very low spike rates, the across-trial variance is much smaller than the mean spike count. (2) The response variance is affected in different ways by the size and the contrast of the stimulus pattern. Whereas, in a given activity class, the variance does not much depend on pattern size, it is significantly larger at low-pattern than at high-pattern contrasts.

One way to explain these results is to relate them to the membrane potential and its fluctuations at the spike initiation zone of the $\mathrm{H} 1$ neuron. These membrane potential fluctuations can be split up into two components. One component is induced deterministically by the stimulus. The other component varies across trials and will be called stochastic. These membrane potential changes are not easily accessible in the $\mathrm{H} 1$ neuron and cannot be systematically varied in an experimental analysis. Therefore, model simulations were performed to analyze what characteristics of the deterministic and the stochastic component may determine the dependence of the spike count variance on the mean spike count. In particular, we investigated how the amplitude and the dynamics of the deterministic and the stochastic component of the membrane potential influence the spike count variance.

The model simulations were based on a phenomenological timedependent threshold model of spike generation that transforms membrane potential fluctuations into sequences of action potentials. The model could be shown in a previous study to be sufficient to account for the time-dependent responses as well as for the reliability of the H1 neuron (Kretzberg et al. 2000). The deterministic component of the membrane potential fluctuations fed into the model was taken from experimental data of another TC in the fly's brain, an HS cell. The stochastic membrane potential component was simulated by band-limited gaussian white noise with a power spectrum adjusted to the electrophysiologically determined membrane potential noise (see Materials and Methods; Kretzberg et al., 2000). HS cells mainly respond with graded changes in their membrane potential, which, in a first approximation, represent the summated postsynaptic potentials of their many local motionsensitive input elements. Apart from their response mode, HS cells respond to motion stimuli in the preferred direction in basically the same way as the H1 cell investigated above (Hausen, 1981; Warzecha, 1994; Haag and Borst, 1997; Warzecha and Egelhaaf, 2000). To analyze the determinants of the spike count variance, we systematically varied in our model simulations the amplitude and the time course of the deterministic membrane potential fluctuations as well as the properties of the membrane potential noise.

\section{Variation of the amplitude of the deterministic membrane potential component}

A given mean membrane potential trace of an HS cell obtained during stimulation with random velocity fluctuations was used in three different ways as input to the model, i.e., either in its original form or scaled in amplitude by a factor of either 0.5 or 1.5 . The properties of the superimposed membrane potential noise were identical for all three versions of the deterministic input component. The resulting spike frequency histograms are modulated over time in a similar way as the corresponding responses of the $\mathrm{H} 1$ cell (data not shown) (but see Kretzberg et al., 2000). When the spike rate, determined in $100 \mathrm{msec}$ time windows, is plotted as a function of the corresponding deterministic membrane potential component, an almost linear relationship between both variables is obtained, irrespective of whether experimental or simulated data are evaluated (Fig. 6A). Virtually the same spike count is obtained for 

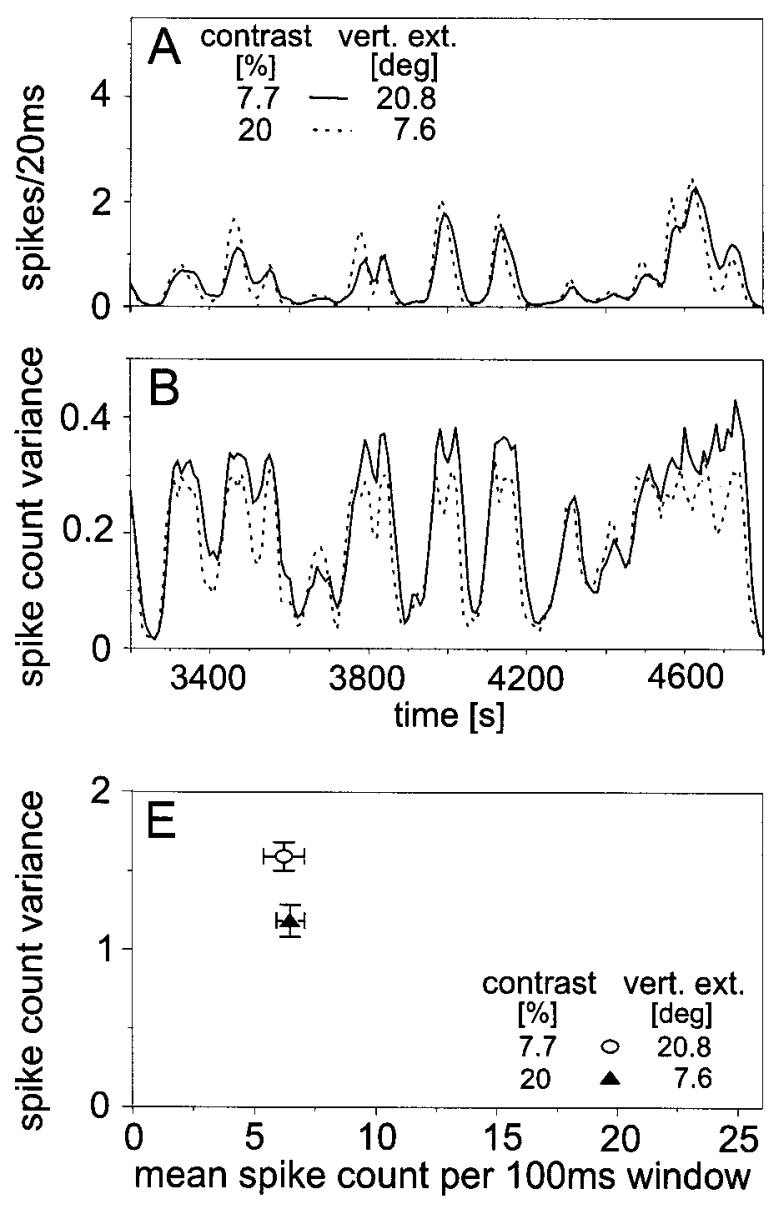
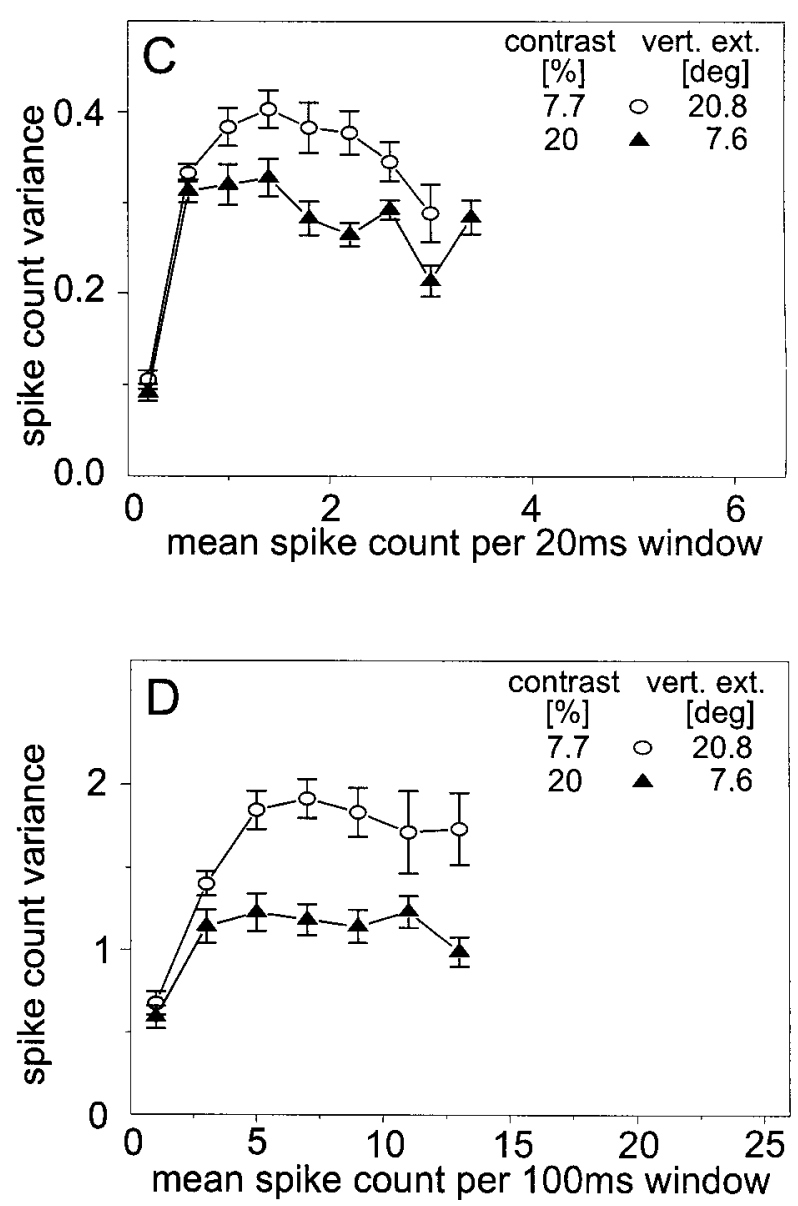

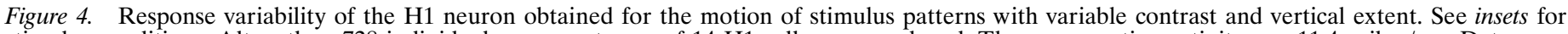

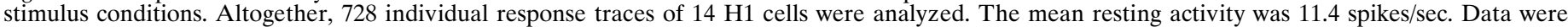

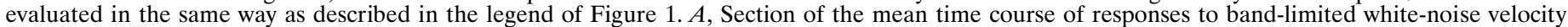

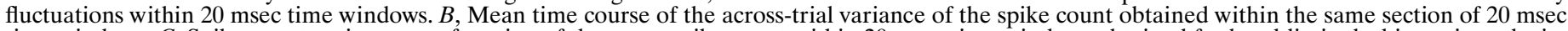

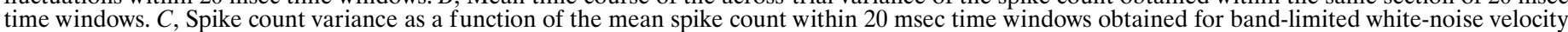

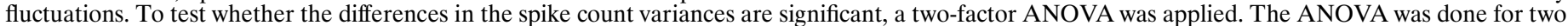

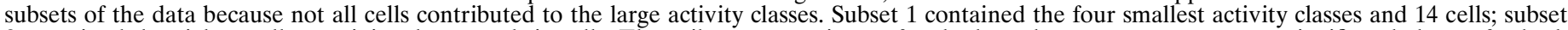

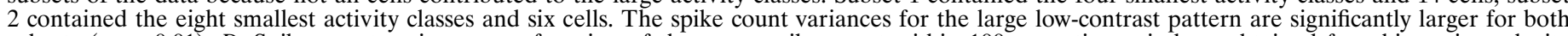

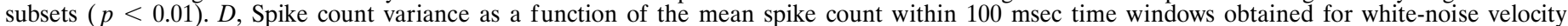

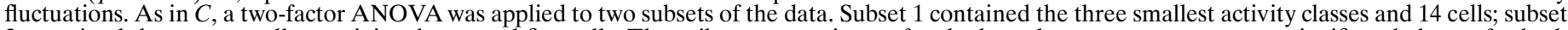

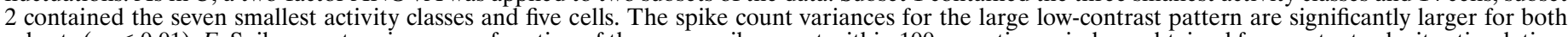

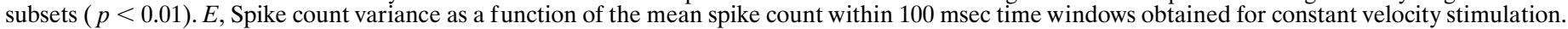

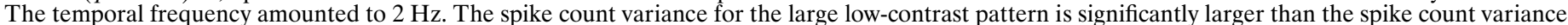

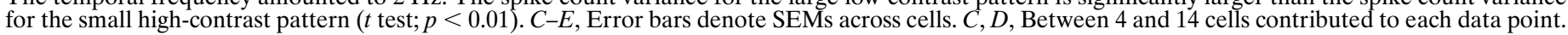

a given amplitude of the deterministic component, independent of the scaling factor. In the simulations, the cell was depolarized by up to $10 \mathrm{mV}$. Fly HS cells do not depolarize much more when recorded in their axon, even during strong visual stimulation. Because of the refractory properties of the model neuron, the spike count will start to saturate when the cell is further depolarized (Fig. 7A). The largest hyperpolarization attained during visual stimulation were approximately $-10 \mathrm{mV}$. In the $\mathrm{H} 1$ cell as well as in the model cell, spikes are elicited even when the deterministic membrane potential component is negative relative to the resting potential $(0 \mathrm{mV})($ Fig. $6 A)$. In the model, such events are primarily attributable to stochastic membrane potential fluctuations occasionally passing the threshold. These results suggest that the spike rate of the $\mathrm{H} 1$ neuron is approximately proportional to the membrane potential at its spike initiation zone for most of the range of postsynaptic potentials elicited during visual stimulation. A linear relationship between membrane potential and spike rate is in accordance with previous experimental results on fly $\mathrm{TCs}$; the spike count in the $\mathrm{H} 1$ neuron and the average membrane potential of the HS cell depend in basically the same way on visual stimulation (Hausen, 1981), and the mean time course of the responses of both cell types is very similar during preferred direction motion
(Kretzberg et al., 2000, their Fig. 2). However, the linear relationship between membrane potential and spike count is in contrast to a strong saturation of spike activity that was hypothesized for retinal ganglion cells even for relatively small depolarizations (Berry and Meister, 1998).

The across-trial variance of the simulated spike count was evaluated as described for the experimental data. The time-dependent variance fluctuates in accordance with the experimental data (data not shown). Analyzing the dependence of the variance on the mean spike count reveals that the variance is low for small spike counts and highest for intermediate activities. The variance is always smaller than the mean spike count apart from the lowest activity class (Fig. 6B). Note that the variance already starts to decrease with increasing activity at $\sim 2$ spikes/20 msec time window (i.e., 100 spikes/sec), when the mean spike count still steeply increases with increasing depolarization. As is the case for the H1 cell, the initial increase in the variance is considerably more pronounced when the spike count variance is evaluated within $20 \mathrm{msec}$ than within $100 \mathrm{msec}$ time windows (Fig. 6, compare $B, C$ ). Interestingly, the spike count variance is not identical for the three inputs of different amplitude, although the properties of the membrane potential noise were identical. This is particularly obvious for 


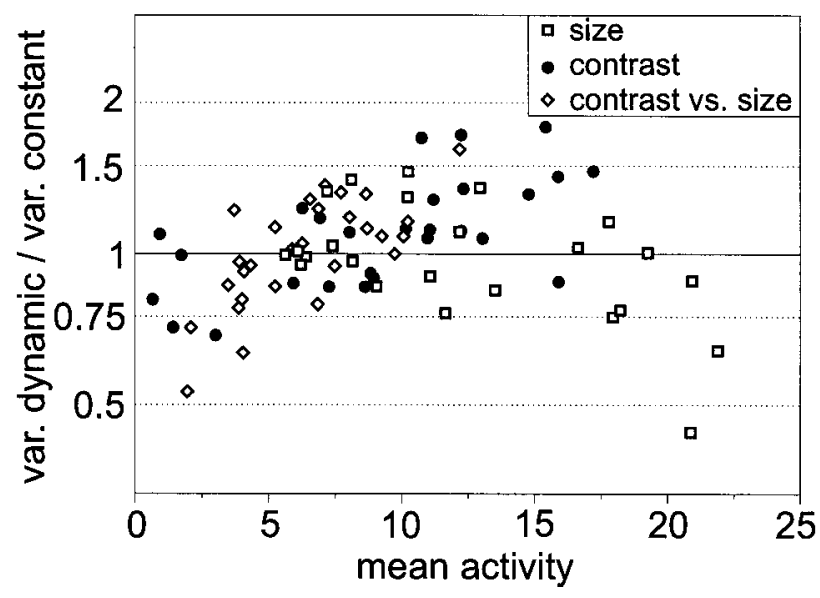

Figure 5. Variability between different $\mathrm{H} 1$ cells and stimulus conditions. The quotient between the mean spike count variance for dynamical and that for constant velocity stimulation is plotted as a function of the mean spike count during constant stimulation. The mean spike count and its variance during constant velocity stimulation were evaluated within 100 msec time windows for each cell and stimulus condition separately, as described in Materials and Methods. To obtain the mean spike count variances for dynamic stimulation, only those $100 \mathrm{msec}$ time windows were taken into account for which the mean activity fell into the same activity range that was covered by constant velocity stimulation. The largest (smallest) mean spike count variance contributing to the figure amounted to 3.14 spikes $^{2} / 100 \mathrm{msec}\left(0.56\right.$ spikes $\left.^{2} / 100 \mathrm{msec}\right)$ for constant stimulation and to 2.70 spikes $^{2} / 100 \mathrm{msec}\left(0.68\right.$ spikes $\left.^{2} / 100 \mathrm{msec}\right)$ for dynamical stimulation. Data are part of those used for Figures 1,2, and 4. Symbols indicate the different data sets. For each cell, two or three data points (depending on the data set) are shown, which were obtained by the different stimulus conditions.

the 100 msec time window (Fig. 6C). The largest membrane potential fluctuations are associated with the smallest spike count variance. Thus, at least for the large time window, the spike count variance differs for the three different input signals, although the membrane potential noise has not changed. These model simulations with physiologically plausible parameters allow us to conclude that the spike count variance is affected, at least to a small extent, by the overall amplitude of the deterministic, stimulus-induced input. Therefore, it is not possible to infer, without further evidence, changes in the stochastic component of the membrane potential from changes in the spike count variance. Merely changing the amplitude of the deterministic membrane potential fluctuations and leaving the stochastic membrane potential fluctuations unaltered may suffice to explain the slight dependence of the spike count variance on stimulus size (Fig. $1 C, D$ ).

\section{Variation of the time course of the deterministic membrane potential component}

To test whether the dynamics of the deterministic membrane potential component affects the variability of the spike output, input signals that differed with respect to their dynamical properties were used as input to the model of spike generation: (1) constant, (2) fluctuating according to the experimental results, or fluctuating (3) twice ("fast membrane potential dynamics") or (4) four times as fast ("very fast membrane potential dynamics"). The latter two stimuli were obtained by compressing the time scale of the signal without changing its amplitude. The dynamics of the membrane potential input does not much affect the spike count variance determined in $20 \mathrm{msec}$ time windows as long as the membrane potential fluctuations are not faster than those elicited by white-noise velocity stimulation (Fig. $7 B$ ). Even constant membrane potentials lead to very similar spike count variances as membrane potential fluctuations with dynamics as found when the cell is stimulated with white-noise velocity fluctuations. Only when the membrane potential fluctuates at least two to four times as fast as has been elicited in motion-sensitive neurons by white-noise velocity fluctuations, the spike count variance decreases to some extent (Fig. $7 B$ ). The spike count variance evaluated within 100 msec time windows does not consistently depend on the membrane potential dynamics (Fig. 7C). This inconsistency might be caused by smoothing out the very fast fluctuations by such relatively large time windows. Neither the variation of the amplitude (Fig. 6) nor the variation of the dynamical properties of the mean membrane potential (Fig. 7) yielded changes of the corresponding spike count variances that are comparable with the experimentally determined ones when pattern contrast was altered (Figs. $2 C, D, 4 C, D$ ).
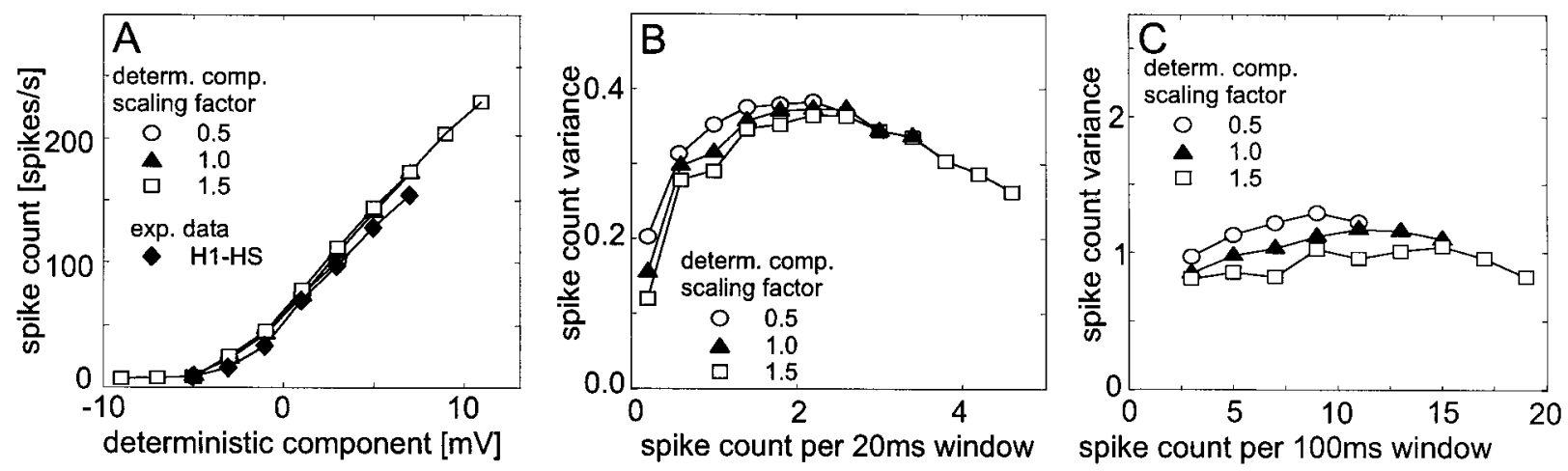

Figure 6. Dependence of the response properties of a simulated spiking neuron on the amplitude of the deterministic membrane potential component. The deterministic component scaled with a factor of 1 corresponds to the unaltered membrane potential fluctuations of a tangential cell to dynamic motion stimulation averaged across 100 trials. It lasted for $2960 \mathrm{msec}$. A membrane potential of $0 \mathrm{mV}$ corresponds to the resting potential of the tangential cell. The amplitude of the deterministic membrane potential component was increased and decreased by $50 \%$ (see insets). The stochastic membrane potential component was fitted to the experimental data (see Materials and Methods). The mean spike count and the spike count variance were determined across 500 individual response traces for each input condition. $A$, Dependence of the spike count on the deterministic membrane potential component for simulated and experimentally determined data (see inset). Note that different symbols superimpose. The mean deterministic component and the mean spike count were determined in $20 \mathrm{msec}$ time windows. The mean deterministic membrane potential was assigned to activity classes with a width of $2 \mathrm{mV}$. Spike counts were averaged if the corresponding mean membrane potential fell into the same activity class. For the experimental data, 100 responses from an $\mathrm{H} 1$ neuron were evaluated. The neuron was stimulated with the same dynamic motion fluctuations as the HS cell used to determine the deterministic response component of the membrane potential. In another recording (data not shown), the mean spike count for each activity class of the membrane potential was slightly larger than that of the model cell. $B$, Spike count variance as a function of the mean spike count within 20 msec time windows. As for the experimental data (see Materials and Methods), the mean spike count was assigned to activity classes with a width of 0.4 spikes per time window. Spike count variances were averaged if the corresponding mean spike count fell into the same activity class. $C$, Spike count variance as a function of the mean spike count within $100 \mathrm{msec}$ time windows. Consecutive time windows overlapped by $90 \mathrm{msec}$. The mean spike count was assigned to activity classes with a width of two spikes per time window. Spike count variances were averaged if the corresponding mean spike count fell into the same activity class. 

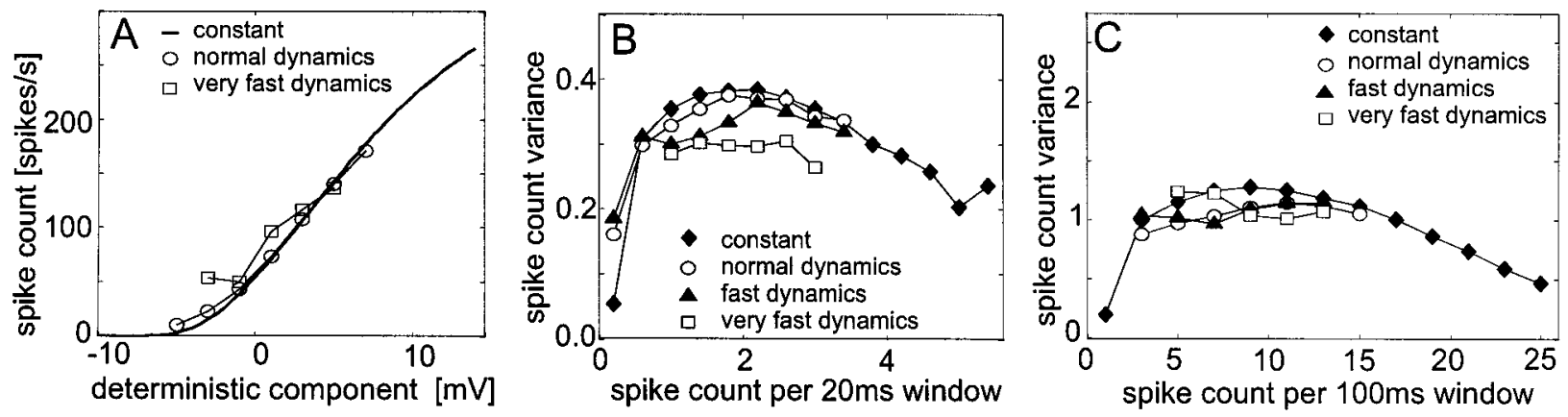

Figure 7. Dependence of the responses of a simulated spiking neuron on the dynamics of the deterministic component of membrane potential. To obtain the constant deterministic component, the membrane potential was set to constant values. To cover the entire response range of a tangential cell, this value was increased in steps of $0.5 \mathrm{mV}$ in subsequent simulations. The deterministic component with normal dynamics was obtained from averaging 100 responses of a tangential cell to band-limited white-noise velocity fluctuations (the same as used for Fig. 6). Faster dynamics were obtained by compressing the time scale of the deterministic membrane potential component by a factor of 2 (fast dynamics) or by a factor of 4 (very fast dynamics). Therefore, the duration of the membrane potential fluctuations that were fed into the model and the corresponding sequences of spike trains were reduced from 2960 msec (normal dynamics) to either $1480 \mathrm{msec}$ (fast dynamics) or $740 \mathrm{msec}$ (very fast dynamics). The parameters of the stochastic component were fitted to the experimental data. Data evaluation and conventions as described in the legend of Figure 6 . For the dynamical membrane potential fluctuations, the mean spike count and the spike count variance were determined across 500 individual response traces for each condition, for the constant membrane potential; 200 response traces were taken. $A$, Dependence of the spike count on the deterministic component of the membrane potential. $B$, Spike count variance as a function of the mean spike count within $20 \mathrm{msec}$ time windows. $C$, Spike count variance as a function of the mean spike count within 100 msec time windows.

\section{Variation of the properties of the membrane potential noise}

So far, all model simulations were obtained by altering the deterministic membrane potential component without modifying the properties of the membrane potential noise. In the following, the properties of the noise are altered while the deterministic component remains unchanged. For the simulations shown in Figure 8, the noise amplitude was either kept as experimentally derived or it was scaled by a factor of 0.5 or 1.5. For the simulations shown in Figure 9, the noise dynamics was altered by compressing ("fast stochastic component") or dilating ("slow stochastic component") the time scale of the experimentally determined noise signals by a factor of 2 but leaves the amplitude constant. When the properties of the membrane potential noise are altered, the mean spike count associated with a given mean membrane potential as well as the spike count variance are affected (Figs. 8,9). The mean spike count corresponding to a given mean membrane potential increases with increasing the amplitude of the noise (Fig. $8 A$ ). Similarly, larger spike counts are obtained for fast than for slowly varying noise (Fig. 9A). Independent of the time window used for the evaluation of the spike count variance, the spike count variance increases considerably with the amplitude of the membrane potential noise (Fig. $8 B, C$ ) or when the noise fluctuates only slowly (Fig. 9B,C). When the membrane potential noise fluctuates faster than has been determined experimentally, the spike count variance decreases slightly. One reason for the increased variability of spike responses for slow stochastic fluctuations may be the following. Under this condition, the spike count in an arbitrary time window can be rather large because the noise lifts the membrane potential beyond the threshold for a relative long time interval, or it can, for the same time window, be rather low because the noise hyperpolarizes the cell. As a consequence, the spike count variance across trials will be large. In contrast, when the noise fluctuates more rapidly, the intervals with high and low activity attributable to noise alternate more rapidly so that the spike count stays statistically at more intermediate values. This leads to a relatively small across-trial variance. The changes obtained in the spike count variance when the properties of the membrane potential noise are altered may well account for the experimental results obtained for different pattern contrasts. This finding suggests that the stochastic membrane potential fluctuations that are the basis for the variability in the spike count of the fly $\mathrm{H} 1$ cell cannot be regarded to add linearly to the deterministic, stimulus-induced component of the membrane potential. Instead, it is suggested that the stochastic
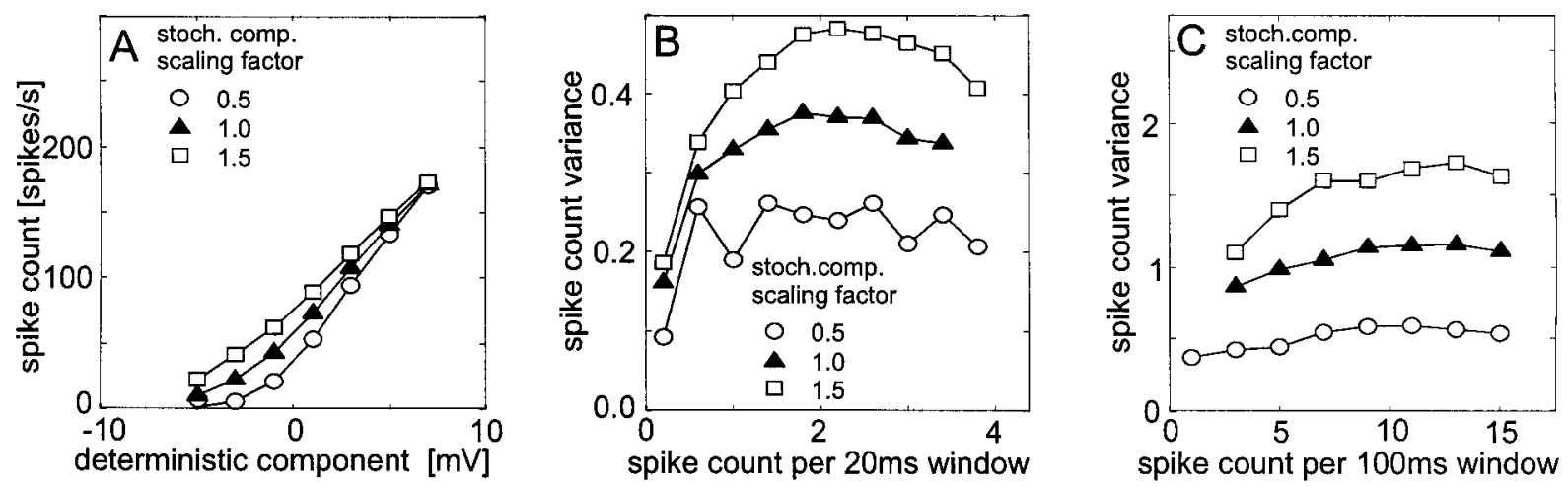

Figure 8. Dependence of the responses of a simulated spiking neuron on the amplitude of the stochastic component of the membrane potential. The stochastic component scaled by a factor of 1 was derived from experimental data. To investigate the influence of the amplitude of the stochastic membrane potential component, it was increased or decreased by $50 \%$ (see insets). The deterministic component was obtained from a tangential cell during stimulation with band-limited white-noise velocity fluctuations (the same as used for Fig. 6). Data evaluation and conventions are as described in the legend of Figure 6. $A$, Dependence of the spike count on the deterministic component of the membrane potential. $B$, Spike count variance as a function of the mean spike count within $20 \mathrm{msec}$ time windows. $C$, Spike count variance as a function of the mean spike count within 100 msec time windows. 

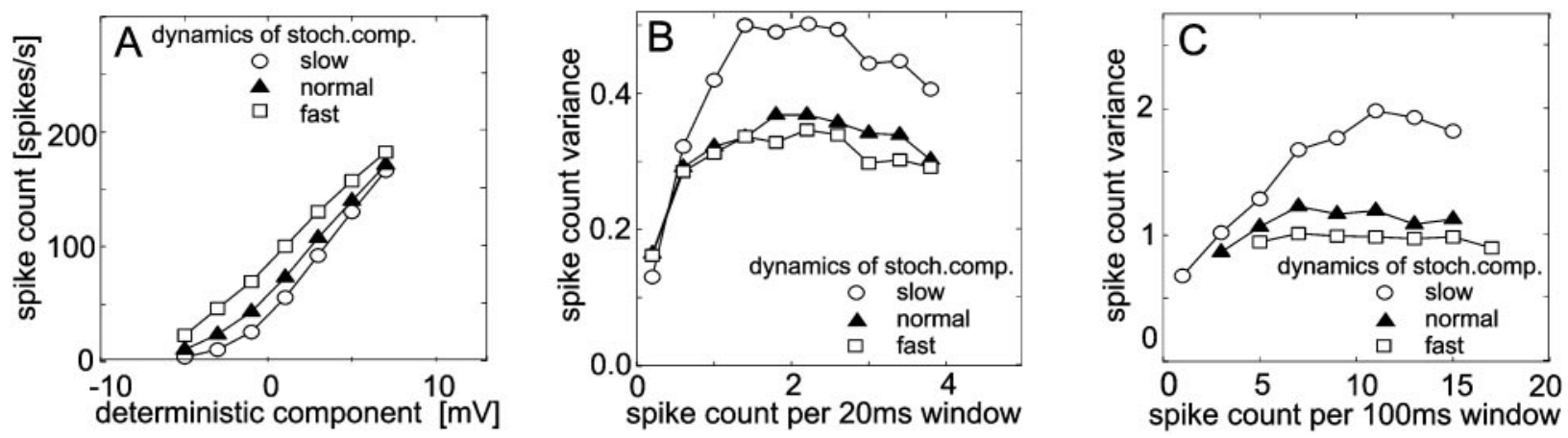

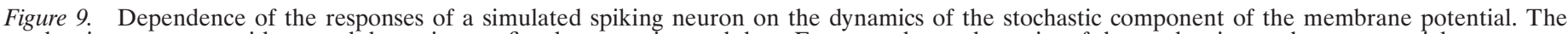

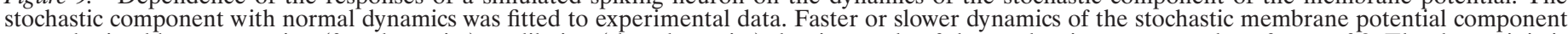

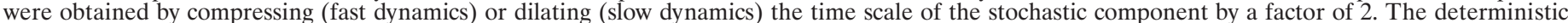

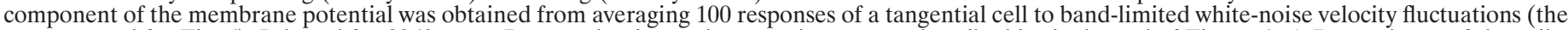

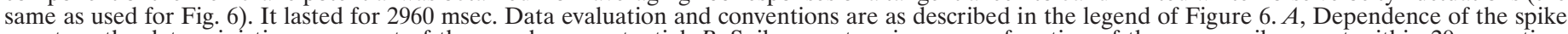

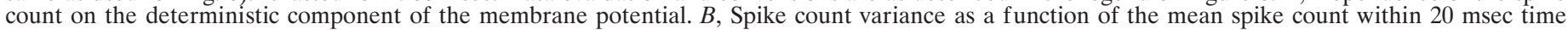
windows. $C$, Spike count variance as a function of the mean spike count within 100 msec time windows.

component decreases in amplitude or speeds up with increasing pattern contrast, inducing a smaller variability of the spike responses.

\section{DISCUSSION}

We investigated how the spike count variance of the $\mathrm{H} 1$ neuron, a motion-sensitive TC in the fly visual system, depends on the stimulus conditions. The following conclusions can be drawn. (1) The spike count variance is much smaller than the mean spike count and does not depend much on the mean activity apart from very low activities. (2) The spike count variance is not determined unambiguously by the activity level of the $\mathrm{H} 1 \mathrm{neu}-$ ron but is also affected by the nature of the stimulus, although differently by different stimulus parameters. Whereas the variance lies in the same range for constant and dynamic stimuli and is not much affected by changes in stimulus size, it increases considerably with decreasing pattern contrast. In accordance with our experimental results, the spike count variance of simulated spike trains does not change much with increasing mean spike activity and is much smaller than the mean spike count apart from the low-activity range. The variance may even decrease in an activity range in which refractoriness does not yet lead to saturation of the mean spike count. Whereas in the model the spike count variance only slightly depends on the dynamics and amplitude of the deterministic membrane potential fluctuations, the membrane potential noise affects the variance more strongly.

\section{Relationship between membrane potential fluctuations and spike count variance}

Our model simulations revealed that there is no unambiguous relationship between a given membrane potential noise and the resulting spike count variance. When the deterministic membrane potential fluctuations onto which the noise superimposes get larger, the spike count variance slightly decreases. A similar dependence was obtained experimentally when pattern size was increased. Therefore, this model result suggests that the membrane potential noise is rather independent of pattern size. Because TCs pool with their dendrite, the outputs of many retinotopically arranged elements (for review, see Egelhaaf and Borst, 1993a; Egelhaaf and Warzecha, 1999), this conclusion indicates that the number of input elements activated by motion stimulation does not markedly influence the amplitude of the membrane potential noise in TCs. The decrease in spike count variance found for an increasing pattern contrast is too pronounced to be explained by the change in amplitude of the deterministic component with pattern contrast. Rather, our model simulations suggest that the membrane potential noise either increases in amplitude or slows down with decreasing pattern contrast.
In addition to the amplitude of the deterministic membrane potential component, also its dynamics was found in the model to affect the spike count variance. When the membrane potential transients get faster, the spike count variance slightly decreases. This modeling result should not be confounded with an earlier claim (de Ruyter van Steveninck et al., 1997) that constant stimuli lead to variances of $\mathrm{H} 1$ cell responses in the range of the mean spike count. In contrast, we found for the $\mathrm{H} 1$ neuron that the spike count variances elicited by dynamical and by constant stimuli are much smaller than the mean spike count, apart from low spike activities (Warzecha and Egelhaaf, 1999). It should be noted that the variance of model cell responses is reduced only slightly by changes in the dynamics of the deterministic membrane potential fluctuations and only if these are much more transient than those that are elicited by white-noise velocity fluctuations as used in the experimental analyses. Hence, the model results are in accordance with our experimental data but in contrast to the conclusion drawn by de Ruyter van Steveninck et al. (1997) (for a detailed discussion of the discrepancies between the studies, see Warzecha and Egelhaaf, 1999). In a recent study, de Ruyter van Steveninck et al. (2000) show responses of an H1 neuron with variances elicited by constant velocity motion that are much smaller than the mean spike count, apart from low spike activities. This finding is in accordance with our data. Nonetheless, in the example shown by de Ruyter van Steveninck et al. (2000), the variance obtained with dynamical stimuli is smaller than that for constant stimuli, a finding which they interpret to support their earlier conclusions. It should be noted, however, that de Ruyter van Steveninck et al. $(1997,2000)$ argue on the basis of only a single example. Both their variances of responses to constant and dynamical stimuli are in the range of what we obtained in individual flies. In our sample of data, there are individual flies with response variances induced by constant velocity stimuli that are larger than those elicited by dynamical stimuli and vice versa. On average, variances lie in the same range for both stimulus dynamics (Fig. 5). Given the great interindividual variability of the $\mathrm{H} 1$ cell responses, no sound quantitative conclusions can be drawn on the basis of data obtained on single examples.

\section{Comparison with other cell types}

In cortical neurons, the spike count variance is usually found to be in the range of the mean spike count (see introductory remarks). Similar to the fly $\mathrm{H} 1$ neuron, the variance of retinal ganglion cells has been reported to be much smaller (Berry et al., 1997). However, there are some discrepancies with respect to the dependence of the response variability of retinal ganglion cells on the activity level and the visual stimuli. In part of the studies, it has been 
concluded that the spike count variance increases with the mean spike count with approximately a power law relationship (Levine et al., 1988, 1992). In other studies, the response variability was found to be essentially independent of the stimulus and the activity level of the cell (Dijk and Ringo, 1987; Troy and Robson, 1992; Croner et al., 1993).

The differences in spike count variance in cortical neurons on the one hand, and in retinal ganglion cells and fly TCs on the other hand, might be attributable to different statistical properties of the membrane potential noise. In our model simulations, the membrane potential noise had a gaussian probability distribution that fitted our experimental results fairly well (Hengstenberg, 1982; Haag and Borst, 1997), although the experimentally determined distributions often slightly deviate in a characteristic manner from a gaussian distribution. In cortical visual interneurons, the membrane potential fluctuations may deviate considerably from a gaussian distribution (Ferster and Carandini, 1996; Azouz and Gray, 1999). The different statistics of membrane potential fluctuations in fly TCs and retinal ganglion cells compared with cortical neurons might be attributable to a different input organization. Whereas TCs as well as retinal ganglion cells receive their major synaptic input from neurons originating from more peripheral processing stages, cortical neurons receive most of their input from feedback connections originating from higher processing stages (Barberini et al., 2000).

\section{Sources of response variability}

Whereas the deterministic component of the membrane potential results from the processing of the changes in light intensity elicited during visual motion, the origin of the stochastic response component is not as clear. The reliability of TCs has been proposed to be limited by photoreceptor noise (de Ruyter van Steveninck, 1986; Bialek et al., 1991). Although this possibility may apply to low light levels, under photopic conditions the synapses between photoreceptors and first-order interneurons contribute considerably to the membrane potential noise of the latter type of cells (Laughlin et al., 1987; Juusola et al., 1994, 1995, 1996). The noise level in neurons of the peripheral visual system depends on the temporal properties and the luminance of the stimulus (for review, see Laughlin, 1989; Juusola et al., 1996). It cannot easily be predicted how these findings relate to the response variability of the TCs. Between the first-order visual interneurons and the TCs, several neurons are interposed (for review, see Hausen and Egelhaaf, 1989; Strausfeld, 1989). Therefore, additional noise is most likely introduced into the system at these processing stages. Irrespective of the precise origin of the membrane potential noise, it is clear from the highly synchronized spike activity of TCs with primarily overlapping receptive fields (Warzecha et al., 1998) that most of the noise is generated peripherally to them. Only a minor part of it is attributable to their input synapses and to ion channel noise at the level of the motion-sensitive cells themselves (Kretzberg et al., 2000).

It may surprise that the spike count variance does not increase with pattern size and thus with the number of activated input channels. It seems most likely that also those elements that are not activated by the motion stimulus contribute considerably to the overall variability. In fact, even without motion stimulation, the noise level in TCs was found to increase with increasing luminance (Hengstenberg, 1982; Warzecha, 1994).

In summary, the membrane potential noise, as it manifests itself at the level of the TCs, cannot be regarded as a random, stimulusindependent variable that linearly adds to the deterministic component of the membrane potential. Rather, the properties of the noise appear to change with pattern contrast. Because in almost all natural habitats the contrast varies across the retinal images, this result has important functional implications. Moreover, data evaluation procedures that rely on additive gaussian noise need to be tested carefully for their applicability.

\section{REFERENCES}

Azouz R, Gray CM (1999) Cellular mechanisms contributing to response variability of cortical neurons in vivo. J Neurosci 19:2209-2223.

Azouz R, Gray CM (2000) Dynamic spike threshold reveals a mechanism for synaptic coincidence detection in cortical neurons in vivo. Proc Natl Acad Sci USA 97:8110-8115.

Barberini CL, Horwitz GD, Newsome WT (2000) A comparison of spiking statistics in motion sensing neurons of flies and monkeys. In: Computational, neural and ecological constraints of visual motion processing (Zanker JM, Zeil J, eds), pp 307-320. Berlin: Springer.

Berry MJ, Meister M (1998) Refractoriness and neural precision. J Neurosci 18:2200-2211.

Berry MJ, Warland DK, Meister M (1997) The structure and precision of retinal spike trains. Proc Natl Acad Sci USA 94:5411-5416.

Bialek W, Rieke F, de Ruyter van Steveninck R, Warland D (1991) Reading a neural code. Science 252:1854-1857.

Britten KH, Shadlen MN, Newsome WT, Movshon JA (1993) Responses of neurons in macaque MT to stochastic motion signals. Vis Neurosci 10:1157-1169.

Buračas GT, Zador AM, DeWeese MR, Albright TD (1998) Efficient discrimination of temporal patterns by motion-sensitive neurons in primate visual cortex. Neuron 20:959-969.

Croner LJ, Purpura K, Kaplan E (1993) Response variability in retinal ganglion cells of primates. Proc Natl Acad Sci USA 90:8128-8130.

de Ruyter van Steveninck R (1986) Real-time performance of a movementsensitive neuron in the blowfly visual system. PhD thesis, Groningen University.

de Ruyter van Steveninck R, Bialek W (1988) Real-time performance of a movement-sensitive neuron in the blowfly visual system: coding and information transfer in short spike sequences. Proc R Soc Lond B Biol Sci 234:379-414.

de Ruyter van Steveninck R, Bialek W (1995) Reliability and statistical efficiency of a blowfly movement-sensitive neuron. Phil Trans R Soc Lond B Biol Sci 348:321-340.

de Ruyter van Steveninck R, Lewen GD, Strong SP, Koberle R, Bialek W (1997) Reproducibility and variability in neural spike trains. Science 275:1805-1808.

de Ruyter van Steveninck R, Borst A, Bialek W (2000) Real-time encoding of motion: answerable question and questionable answers from the fly's visual system. In: Computational, neural and ecological constraints of visual motion processing (Zanker JM, Zeil J, eds), pp 279-306. Berlin: Springer.

Dijk BW van, Ringo JL (1987) The variation in the light responses of carp retinal ganglion cells is independent of response amplitude. Vision Res 27:493-497.

Eckert H (1980) Functional properties of the H1-neurone in the third optic ganglion of the blowfly, Phaenicia. J Comp Physiol 135:29-39.

Egelhaaf M, Borst A (1993a) A look into the cockpit of the fly: visual orientation, algorithms, and identified neurons. J Neurosci 13:4563-4574.

Egelhaaf M, Borst A (1993b) Movement detection in arthropods. In: Visual motion and its role in the stabilization of gaze (Wallman J, Miles FA, eds), pp 53-77. Amsterdam: Elsevier.

Egelhaaf M, Reichardt W (1987) Dynamic response properties of movement detectors: theoretical analysis and electrophysiological investigation in the visual system of the fly. Biol Cybern 56:69-87.

Egelhaaf M, Warzecha A-K (1999) Encoding of motion in real time by the fly visual system. Curr Opin Neurobiol 9:454-460.

Ferster D, Carandini M (1996) Spontaneous fluctuations in membrane potential of complex cells in visual cortex. Soc Neurosci Abstr 22:198.4.

Haag J, Borst A (1997) Encoding of visual motion information and reliability in spiking and graded potential neurons. J Neurosci 17:4809-4819.

Hausen K (1981) Monocular and binocular computation of motion in the lobula plate of the fly. Verh Dtsch Zool Ges 74:49-70.

Hausen K, Egelhaaf M (1989) Neural mechanisms of visual course control in insects. In: Facets of vision (Stavenga D, Hardie R, eds), pp 391-424. Berlin: Springer.

Hengstenberg R (1982) Common visual response properties of giant vertical cells in the lobula plate of the blowfly Calliphora. J Comp Physiol 149:179-193.

Johnston D, Wu M-S (1995) Foundations of cellular neurophysiology. Cambridge, MA: MIT.

Juusola M, Kouvalainen E, Järvilehto M, Weckström M (1994) Contrast gain, signal-to-noise ratio and linearity in light-adapted blowfly photoreceptors. J Gen Physiol 104:593-621.

Juusola M, Uusitalo RO, Weckström M (1995) Transfer of graded potentials at the photoreceptor-interneuron synapse. J Gen Physiol 103:117-148.

Juusola M, French AS, Uusitalo RO, Weckström M (1996) Information processing by graded-potential transmission through tonically active synapses. Trends Neurosci 19:292-297.

Kretzberg J, Egelhaaf M, Warzecha A-K (2000) Membrane potential fluctuations determine the precision of spike timing and synchronous activity: a model study. J Comput Neurosci, in press.

Laughlin SB (1989) Coding efficiency and design in visual processing. In: Facets of vision (Stavenga DG, Hardie RC, eds), pp 213-234. Berlin: Springer. 
Laughlin SB, Howard J, Blakeslee B (1987) Synaptic limitations to contrast coding in the retina of the blowfly Calliphora. Proc R Soc Lond B Biol Sci 231:437-467.

Levine MW, Zimmerman RP, Carrion-Carire V (1988) Variability in responses of retinal ganglion cells. J Opt Soc Am A 5:593-597.

Levine MW, Cleland BG, Zimmerman RP (1992) Variability of responses of cat retinal ganglion cells. Vis Neurosci 8:277-279.

Reinagel P, Reid RC (2000) Temporal coding of visual information in the thalamus. J Neurosci 20:5392-5400.

Strausfeld NJ (1989) Beneath the compound eye: neuroanatomical analysis and physiological correlates in the study of insect vision. In: Facets of vision (Stavenga DG, Hardie RC, eds), pp 317-359. Berlin: Springer.

Tolhurst DJ, Movshon JA, Dean AF (1983) The statistical reliability of signals in single neurons in cat and monkey visual cortex. Vision Res 23:775-785.

Troy JB, Robson JG (1992) Steady discharges of X and Y retinal ganglion cells of cat under photopic illuminance. Vis Neurosci 9:535-553.
Vogels R, Spileers W, Orban GA (1989) The response variability of striate cortical neurons in the behaving monkey. Exp Brain Res 77:432-436.

Warzecha A-K (1994) Reliability of neuronal information processing in the motion pathway of the blowflies Calliphora erythrocephala and Lucilia cuprina. $\mathrm{PhD}$ thesis, Universität Tübingen.

Warzecha A-K, Egelhaaf M (1997) How reliably does a neuron in the visual motion pathway of the fly encode behaviourally relevant information? Eur J Neurosci 9:1365-1374.

Warzecha A-K, Egelhaaf M (1999) Variability of neural responses during constant and dynamical stimulation. Science 283:1927-1930.

Warzecha A-K, Egelhaaf M (2000) Neuronal encoding of visual motion in real-time. In: Computational, neural and ecological constraints of visual motion processing (Zanker JM, Zeil J, eds), pp 239-277. Berlin: Springer.

Warzecha A-K, Kretzberg J, Egelhaaf M (1998) Temporal precision of encoding of motion information by visual interneurons. Curr Biol 8:359-368. 